\title{
Forecasting: necesidades desde la educación contable para su abordaje
}

\author{
Evelyn Ivonne Díaz Montaño ${ }^{1}$ \\ Jorge Suárez Tirado
}

Recibido: 25 de octubre de 2014

Aprobado: 14 de noviembre de 2014

\begin{abstract}
Díaz, E. \& Suárez, J. (2014). Forecasting: necesidades desde la educación contable para su abordaje. Activos 23, 197-226
\end{abstract}

Clasificación JEL: M49

\section{Resumen}

El forecasting constituye un instrumento que busca pronosticar los resultados de una o varias operaciones en las organizaciones, desde diferentes áreas estratégicas que, al combinarse, pueden generar efectos en los estados financieros presentes y futuros. Esta posibilidad está vinculada al cumplimiento de los objetivos estratégicos de la organización, ya que el análisis de estos efectos proyectados se puede confrontar con la viabilidad de las operaciones que se desarrollan o que se planean desarrollar.

El diseño e implementación de instrumentos como el forecasting requieren algo más que el dominio de métodos de control; requieren creatividad

1 Contadora pública, (C) Optante a Maestra en Medio Ambiente y Desarrollo de la Universidad Nacional de Colombia. Especialista en Gerencia de los Recursos Naturales de la Universidad Distrital. Docente de tiempo completo de la Universidad Santo Tomás.

2 Contador público. Magíster en Administración de la Universidad Nacional de Colombia. Docente de tiempo completo de la Universidad Jorge Tadeo Lozano. 
y recursividad a la hora de articular los procesos estratégicos en las organizaciones. Este tema se desenvuelve, propiamente, dentro del marco de la toma de decisiones bajo incertidumbre, por lo tanto, los requerimientos desde la educación contable en las áreas de control toman relevancia desde la perspectiva interdisciplinar.

En ese sentido, resulta adecuado en las asignaturas de contabilidad de gestión el abordaje de temas como el forecasting y las diferentes técnicas que se utilizan como apoyo a los procesos de predicción y toma de decisiones en las compañías, las cuales provienen principalmente de la estadística, la econometría, la administración de operaciones y la investigación de operaciones.

\title{
Palabras clave
}

Forecast, control, incertidumbre, gestión, control, educación.

\section{Díaz, E. \& Suárez, J. (2014). Forecasting: From accounting education needs to address them. Activos 23, 197-226}

\begin{abstract}
The forecast is an instrument made to predict the results of one or more operations from strategic areas which, when combined, can produce effects on the current and future financial statements. This possibility is linked to the fulfilment of the organization's strategic objectives, as the analysis of these predicted effects can be confronted with the viability of the operations carried out or due to be developed.

The design and implementation of tools such as forecasting require more than just the domain of control methods; they require creativity and resourcefulness when articulating the strategic processes within organizations. This topic unfolds itself within a decision-making framework under
\end{abstract}


uncertainty. Therefore, the requirements for accounting education in control areas become relevant from an interdisciplinary perspective.

In this regard, within the subjects of management accounting, it is appropriate to address topics such as forecasting and the various techniques that are used to support the prediction processes within companies, which are mainly from statistics, econometrics, operations management and operations research.

\section{Keywords}

Forecasting, control, uncertainty, management, education.

\section{Díaz, E. \& Suárez, J. (2014). Forecasting: Besoins de l'education comptable pour son approche. Activos 23, 197-226}

\section{Résumé}

Le forecasting constitue un outil qui a pour but de prévoir les résultats d'une ou plusieurs opérations dans les entreprises, à partir des différents domaines stratégiques qui, au moment d'être combiné, peuvent avoir des conséquences sur les états financiers actuels et à venir. Cette probabilité est liée à l'accomplissement des objectifs stratégiques de l'entreprise, puisque l'analyse de ces effets prévus peut se confronter avec la viabilité des opérations qui se déroulent ou qui sont prévues.

L'élaboration et la mise en œuvre d'outils tel que le forecasting nécessitent bien plus que la maîtrise de méthodes de contrôle; elles ont besoin de créativité y d'ingéniosité au moment d'articuler les processus stratégiques dans les entreprises. Ce thème se déroule, particulièrement, dans le cadre de la prise de décisions sous incertitude, donc, les exigences depuis l'éducation comptable dans les domaines de contrôle prennent de l'importance du point de vue pluridisciplinaire. 
Dans ce sens, il est approprié d'aborder des thèmes tels que le forecasting dans les cours de comptabilité, ainsi que les différentes techniques qui s'utilisent comme soutien aux processus de prédiction et de prise de décision dans les entreprises, lesquelles proviennent principalement de la statistique, l'économétrie, la gestion des opérations et la recherche des opérations.

\section{Mots clés}

Forecast, contrôle, incertitude, gestion, contrôle, éducation. 


\section{Introducción}

Los procesos de pronóstico han sido apropiados especialmente por las compañías multinacionales, las cuales sitúan la estrategia como el nodo central a través del cual se estructuran los negocios y se materializan los objetivos organizacionales. En Colombia, aunque se ha venido trabajando en este tipo de informes de pronóstico aproximadamente durante la última década, no se cuenta oficialmente con un manual o publicación que actúe como guía o modelo para su elaboración en el escenario de la toma de decisiones bajo incertidumbre, razón por la cual se hace necesaria la investigación y pronunciamiento por parte de la comunidad académica al respecto.

El diseño e implementación de instrumentos de pronóstico, requieren de algo más que el dominio de métodos de control; exige creatividad y recursividad a la hora de articular los procesos estratégicos en las organizaciones. Estos requerimientos van desde la teoría general del costo hasta las consideraciones teóricas de la contabilidad, es decir que desde lo disciplinar, la apropiación metodológica y conceptual de la contabilidad es requerida para realizar un pronóstico.

Se necesita una comprensión total de la organización y de la información de carácter sistémico, debido a las implicaciones de los pronósticos sobre el agregado de la compañía. Sin embargo, no bastan los conocimientos desde lo instrumental para el abordaje de una herramienta planteada desde la estrategia, ya que uno de sus principales retos radica precisamente en su diseño.

Se requiere una mayor formación interdisciplinar, según señala Gómez (2003) respecto a las potencialidades de investigación en Colombia: por un lado, el investigador puede manejar instrumentos ortodoxos de la economía y la administración, tales como la microeconomía, la economía de empresa y la teoría organizacional, aunado esto al manejo de herramientas estadísticas y econométricas. Por el otro, el investigador debe estar capacitado con herramientas de análisis desde la sociología, la economía política, la ecología, el derecho y las perspectivas heterodoxas de la administración de organizaciones y de la economía, entre otras. 
Es interesante analizar esta relación, ya que puede existir una tendencia a vincular las necesidades de interdisciplinariedad en la educación contable con temas de reflexión en torno al conocimiento.

De acuerdo con lo anterior, el presente documento plantea, por un lado, la generación de un concepto teórico sobre el forecasting, el cual se ha venido trabajando en una etapa preliminar. Por el otro lado, evidencia los principales requisitos para su abordaje conceptual y metodológico desde la disciplina contable y particularmente desde la contabilidad de gestión, describiendo brevemente algunas técnicas para elaborar pronósticos.

La presente investigación se enmarca dentro de una perspectiva analítica e interpretativa. Para su desarrollo, se revisan diferentes visiones sobre el forecasting. El vínculo existente entre el desarrollo y avance del conocimiento contable y la estrategia es cada vez más estrecho. Una de las principales ventajas que se identifican fácilmente desde la contabilidad para la gestión en los niveles estratégicos radica en la apropiación de la instrumentalización requerida para diseñar, generar e interpretar información.

\section{El forecasting}

La contabilidad de costos se ha desarrollado principalmente a través de la evolución de los diferentes sistemas de costeo, donde el objetivo es determinar los desembolsos en que se incurre en la fabricación y comercialización de bienes o en la prestación de servicios. En busca de trascender estos límites, los desarrollos en contabilidad de gestión se han orientado hacia el proceso de toma de decisiones a partir de la información que generan los sistemas de costos.

Es en este punto donde viene a hacer presencia el forecasting, entendido como el proceso de pronóstico que, a partir de información combinada de variables internas y datos macroeconómicos, apoya las áreas estratégicas de las organizaciones y favorece el proceso de toma de decisiones (Díaz y Suárez, 2014). 
De acuerdo con Horngren, Datar y Foster (2007), el éxito en una compañía depende de la creación de valor para los clientes y de la diferenciación con sus competidores; la estrategia de la entidad se fundamenta en la identificación de cómo lograr esta tarea. Diseñar las estrategias necesarias para lograr la situación descrita constituye una tarea que se lleva a cabo entre los contadores administrativos y los gerentes.

Uno de los aspectos clave por tener en cuenta al momento de tomar decisiones es la incertidumbre que acompaña al futuro. Para enfrentarla, se requiere contar con información adecuada sobre posibles escenarios que vendrán en tiempo posterior, para construir estrategias y poder elegir entre las diferentes alternativas disponibles con mayor seguridad y precisión. Courtney, Kirkland y Viguerie (1997) consideran que subestimar la incertidumbre puede llevar a la elaboración de estrategias que no sirvan ni para defenderse de ella ni para enfrentarla. Hamel y Prahalad (1994) afirman que para estar a la vanguardia en cuanto a los cambios que presenta un sector de la economía, es necesario que los altos directivos entiendan que un aspecto clave es competir por el futuro.

El alcance de los objetivos de la compañía se verá afectado de manera transversal por los instrumentos idóneos para su consecución, de tal forma que ya no será suficiente la apropiación de una metodología; los aportes de quienes diseñan estas herramientas se concentrarán en su capacidad de vincular su conocimiento a las posibilidades que pueda arrojar la organización desde cualquier nivel. Es importante tener en cuenta la posibilidad de combinar diversos tipos de información; además, el no descuidar los aspectos éticos vinculados debe ser una característica propia del proceso de toma de decisiones bajo incertidumbre.

Desde los retos de la creación de informes que permitan analizar efectos sobre el cumplimiento de los objetivos corporativos, se reconoce que las formas de representación de la información pueden ser diversas, por lo tanto, los criterios de temporalidad, trazabilidad y control no se encuentran reflejados a través de una construcción rígida y replicable en todos los casos de la misma forma. 
Como señalan Díaz \& Suárez (2014), actualmente uno de los instrumentos implementados al interior de las empresas como parte del diseño de una estrategia es el forecasting o informes de predicción que pueden ser aplicados con diferentes intencionalidades, temporalidad, trazabilidad y control, siendo estas sus principales características. Estas estructuras no tienen un patrón rígido de comportamiento, y en su mayoría forman parte de la apropiación de conocimiento particular por parte de cada organización.

El punto de partida para referirse a la generación de un forecasting es, como lo señalan Shahzad, Luqman, Khan y Shabbir (2012), la relación entre la cultura organizacional y su impacto en el desempeño de la organización, al encontrarse directamente vinculada con el logro de los objetivos de la organización. Es determinante este tipo de consideración, ya que para la elaboración de un pronóstico, la organización se encuentra determinada por su concepto y no por su objeto social, es decir, las estrategias encaminadas al logro de los objetivos reflejan la esencia de la organización más allá de las actividades de producción.

El forecasting puede representar uno de los ejemplos que vinculan la planeación y el control. Según Horngren et al. (2007, p. 7), "La planeación comprende la selección de las metas de la organización, la predicción de resultados de acuerdo con varias alternativas para alcanzar esas metas". La herramienta de planeación más importante es un presupuesto, el cual es la expresión cuantitativa de un plan de acción propuesto por la administración, y constituye una ayuda para coordinar aquello que debe hacerse con el fin de poner el plan en marcha.

El control consiste en emprender acciones que pongan en marcha las decisiones de planeación, decidir cómo evaluar el desempeño y proporcionar retroalimentación que ayudará a la toma de decisiones a futuro (Horngren, et al., 2007). A partir de la anterior aproximación hacia un concepto de forecasting, se pueden asociar los siguientes aspectos:

- Las construcciones iniciales del forecasting se encuentran direccionadas hacia el pronóstico de ventas, siendo este el principal insumo 
para su consolidación, debido a la cantidad de variables que afecta. Sin embargo, existen otros factores que deben considerarse, especialmente aquellos relacionados con la estructura de costos fijos de la compañía.

- Un forecasting puede construirse para analizar efectos diferentes al pronóstico del resultado operacional, por ejemplo, para pronosticar el flujo de caja a través del movimiento de partidas de balance.

- Si se tiene en cuenta que este tipo de informes no se encuentran regulados, ya que obedecen a una estructura interna y a los intereses de cada organización, el número de variables que se puede abordar depende de las necesidades o criterios de interés por evaluar, característica que refuerza la importancia del diseño del instrumento que, de fondo, evidencia la capacidad para realizar las preguntas requeridas por parte de quienes lo estructuran.

- El elemento diferenciador de este tipo de informes se encuentra determinado por el cruce de diferentes subinformes de distintas áreas producto de la operación normal de la organización, los cuales se convierten en el soporte de seguridad de la probabilidad de ocurrencia de los pronósticos; por lo tanto, debe existir una gran apropiación de conocimiento en torno al funcionamiento y dinámicas de la organización.

- El efecto deseado, en concordancia con los objetivos estratégicos, puede definir también la construcción del informe, por lo tanto, se puede percibir que tiene un componente subjetivo desde el control y la gerencia, que se operativiza a medida que se construye, mediante los informes soporte.

- Condensa una gran cantidad de información de diferentes áreas y logra vincular información externa a la compañía, incorporándola a los datos internos. Un forecasting puede llegar a tener entre veinte y 
setenta informes de soporte, dependiendo del tipo de organización o del efecto que se quiera examinar.

- Posee la característica de servir como instrumento de evaluación y control en el mediano y largo plazo.

Cabe indicar que el forecasting es un instrumento de libre construcción por parte de la organización y no posee unos criterios estrictos de elaboración; sin embargo, la premisa de estos pronósticos es la articulación con los objetivos estratégicos de la organización, para la optimización del beneficio particular. De tal forma, la información metodológica acerca de la consolidación del forecasting ha sido apropiada y acuñada en el interior de algunas organizaciones, desde las áreas de estrategia, de acuerdo a sus necesidades particulares.

Metodológicamente, la construcción de este instrumento de pronóstico inicia con un informe intermedio de presupuesto, que, en términos de alcance, es elaborado para el largo y el mediano plazo, y culmina con un forecasting que se crea para el año corriente. Conforme con los resultados obtenidos luego de diferentes entrevistas en el sector manufacturero en Bogotá, el forecasting puede estar diseñado de la siguiente forma.

\section{Presupuesto de largo plazo}

Pronostica los resultados para un lapso de cuatro a cinco años; se puede basar en supuestos para su elaboración, específicamente análisis sectoriales, indicadores macroeconómicos, estudios de mercado y datos estadísticos. Esta información, combinada y confrontada con los datos reales arrojados en informes como el estado actual de ventas, comportamiento de proveedores e indicadores de gestión, puede ser una base para articular las proyecciones de todas las variables del negocio - industria, participación de mercado, inflación, tasas de interés, ejecución de proyectos, etc.-一. 


\section{Presupuesto de mediano plazo}

Se elabora en el año anterior al que se quiere pronosticar. Al igual que el presupuesto de largo plazo, toma datos reales y realiza proyecciones un poco más detalladas, a través del pronóstico de variables macroeconómicas, pero también específicas. Suponiendo que el contexto de la organización es una fábrica de paletas, en el pronóstico de largo plazo se puede establecer una cantidad de ventas proyectada en 50.000 paletas en el año siguiente, pero en el de mediano plazo, se puede establecer que se venderá ese volumen repartido en las marcas $\mathrm{X}, \mathrm{Y}, \mathrm{Z}$ por cada mes, lo cual supone un nivel de desagregación y precisión mayor.

El escenario habitual para preparar el pronóstico de mediano plazo es agosto, luego de los habituales cierres semestrales. El motivo principal es la existencia de datos más claros desde los referentes sectoriales, macroeconómicos e internos del comportamiento combinado de datos externos e internos. A partir de este pronóstico se deben generar simulaciones con base en cada mes que se vuelva real, es decir, base septiembre real, base octubre real.

Dentro de las prioridades establecidas por las áreas de gerencia, se indica que en este ejercicio es clave generar escenarios de sensibilidad sobre factores como la tasa de cambio, la industria y la mezcla de ventas. Este análisis se efectúa en cada caso particular para explicar las variaciones frente a los supuestos iniciales.

\section{Forecasting}

Se construye mensualmente, con un nivel de desagregación y detalle más amplio que el de los presupuestos de mediano y largo plazo - proyecta los meses del año que faltan, uno a uno-. Siguiendo el ejemplo de la fábrica de paletas, el primer presupuesto que se establece es vender 50.000 unidades, el segundo especifica qué marcas componen el volumen, y el tercero debe indicar en qué sabores, en qué ciudades, por qué canales de distribución, etc. 


\section{Posibilidades de evaluación frente al costo}

A partir de la metodología de preparación del forecasting se evidencia la posibilidad de comparar los presupuestos y pronósticos de operaciones con la ejecución real de cada mes, identificando de esta manera el error de pronóstico y generando el escenario para tomar acciones sobre la marcha, en caso de detectar diferencias. Sin embargo, esta posibilidad plantea un reto desde lo ético, para el caso del control de gestión, ya que los escenarios creados tienen una tendencia a presentar variaciones, y el propósito de eliminarlas requiere afinar y articular todos los esfuerzos y áreas de la organización para lograr las metas proyectadas.

Otra posibilidad de resultado para la evaluación es la comparación frente al presupuesto de mediano plazo, para ver si los supuestos iniciales se encuentran bien planteados. Puntualmente, se podría revisar en un forecasting de estado de resultados si mes a mes se ha vendido el volumen planteado y cuál es la relación con los productos que son más rentables, o analizar los que están generando resultados negativos, a pesar de vender el mismo volumen.

El forecasting permite evaluar el comportamiento de los centros de costo respecto a los ahorros propuestos o las ejecuciones programadas, para en un análisis más profundo, establecer si las disminuciones que se reflejan en un mes constituyen efectivamente ahorros o reprogramaciones del gasto. En el caso de un forecasting de balance general se puede determinar si los flujos de caja han sido debidamente ejecutados, esto a través del comportamiento esperado.

Finalmente estos cruces de información a través del forecasting pueden entregar un análisis de valor si logran establecer las relaciones de partida del estado de resultados y el balance general, con el fin de determinar si los flujos y los resultados están siendo generados por las actividades correctas o no.

De tal forma existen diferentes usos y aplicaciones de esta herramienta; las posibilidades y alcances del análisis dependen entonces del nivel de 
conocimiento del negocio en particular, y la articulación de estos informes con los objetivos corporativos. Es en este punto donde la finalidad del forecasting, aunque es un instrumento que se alimenta desde lo operativo, pertenece a las áreas de estrategia de la compañía.

Una vez se ha realizado una descripción general sobre el forecasting, a continuación se exponen algunos elementos que buscan impulsar su enseñanza en los programas de formación universitaria en materia contable, específicamente en el área de contabilidad de gestión.

\section{Algunas necesidades desde la educación contable para el abordaje del forecasting}

Como se ha descrito en líneas anteriores, el forecasting realiza aportes a la toma de decisiones y, con ello, a la materialización de la estrategia organizacional, tomando elementos desde la teoría general del costo hasta las consideraciones teóricas de la contabilidad.

Si queremos seguir avanzando en la realización de aportes que permitan potencializar el rol de la contabilidad de gestión en las organizaciones, un tema emergente son los informes de forecasting; de allí la importancia de su estudio.

Tatikonda (citado en Cable, Healy \& Mathew, 2009) afirma que hoy los contadores de gestión gastan menos tiempo en preparar reportes estandarizados y más tiempo analizando, interpretando y suministrando información para la toma de decisiones. Los contadores de gestión se están involucrando más en todos los aspectos de las operaciones diarias de los negocios. Para Ahadiat (citado en Cable, et al., 2009), las facultades de contaduría tienden a enfatizar en temas tradicionales que no son necesariamente importantes para los negocios contemporáneos.

Böer (citado en Cable, et al., 2009) sugiere de manera relacionada, que los libros de texto necesitan poner más énfasis sobre la toma de decisiones, 
los análisis de flujo de efectivo y la industria de los servicios, es decir orientarse más hacia los tomadores de decisiones en la práctica; y en un sentido lapidario, señala que los libros de texto cubren temas que los profesores de contabilidad encuentran interesantes, en lugar de problemas que los directores de contabilidad encuentran importantes.

La tabla 1 presenta una síntesis de las principales aplicaciones del forecasting en las organizaciones.

Tabla 1. Principales aplicaciones del forecasting en las organizaciones

\begin{tabular}{|c|c|}
\hline Áreas & Variables que se pueden pronosticar \\
\hline $\begin{array}{l}\text { Planeación y control de } \\
\text { operaciones }\end{array}$ & $\begin{array}{l}\text { Ventas, inventarios, producción, líneas de producto, } \\
\text { ingreso a un nuevo mercado, ¿qué, cuándo y dónde } \\
\text { producir?, precios, disponibilidad futura de insumos. }\end{array}$ \\
\hline Mercadotecnia & $\begin{array}{l}\text { Fijación de precios, vías de distribución, gastos de } \\
\text { publicidad. }\end{array}$ \\
\hline Economía & $\begin{array}{l}\text { PIB, desempleo, consumo, inversiones, nivel de precios, } \\
\text { tasa de interés, política monetaria y fiscal, planeación } \\
\text { estratégica, etapas del ciclo económico (contracción- } \\
\text { expansión), situación futura del mercados de acciones } \\
\text { (alza-baja), estado futuro del mercado cambiario. }\end{array}$ \\
\hline Especulación & $\begin{array}{l}\text { Rendimientos de las acciones y bonos, tasa de interés, } \\
\text { tasa de cambio, precios. }\end{array}$ \\
\hline $\begin{array}{l}\text { Administración del riesgo } \\
\text { financiero }\end{array}$ & Volatilidad en el rendimiento de las acciones. \\
\hline Planeación de la capacidad & $\begin{array}{l}\text { Capacidad suficiente pero no excesiva, tendencias en } \\
\text { tamaño y participación en el mercado, efectos cíclicos } \\
\text { o estacionales, comportamiento de depreciación para la } \\
\text { fábrica y la tecnología. }\end{array}$ \\
\hline $\begin{array}{l}\text { Presupuestos empresariales } \\
\text { y gubernamentales }\end{array}$ & Ingresos y desembolsos. \\
\hline Demografía & $\begin{array}{l}\text { Población, edad, sexo, raza, gastos en salud. } \\
\text { Infraestructura, seguridad social, asistencia social, } \\
\text { nacimiento, muertes, migración, inmigración. }\end{array}$ \\
\hline Manejo de crisis & $\begin{array}{l}\text { Probabilidad de insolvencia, devaluación de la moneda, } \\
\text { golpes militares. }\end{array}$ \\
\hline
\end{tabular}

Fuente: elaboración propia con base en Diebold (1999, pp. 1-3). 
De acuerdo con Ahadiat (citado en Cable et al., 2009), el medio ambiente es cambiante $\mathrm{y}$, de la mano de estas dinámicas, los negocios también se encuentran en constante transformación como consecuencia de la globalización, el aumento de las tecnologías de la información, nuevas formas de gestión en el gobierno corporativo y otras más. Debido a lo anterior, las organizaciones requieren disponer de nueva información que les permita tomar decisiones en este tipo de entornos, caracterizados por la presencia de riesgos, así como de distintos niveles de incertidumbre.

Bauman (2013) retoma el tema señalando cómo las fuerzas determinantes de la forma en la que actúan las personas y las organizaciones en estos tiempos fluyen en el espacio global, y aun cuando las instituciones políticas sigan en general atadas al suelo, seguirán siendo globales. En ese sentido, resulta fundamental la identificación y estudio de formas emergentes de información que contribuyan a la materialización de las estrategias corporativas que aún, desde lo local, respondan a los desafíos globales.

El contador, al verse involucrado cada día de manera más directa en todos los aspectos de las operaciones organizacionales, tendrá la responsabilidad no solo de analizar los informes, sino también de ser el diseñador de los mismos, tarea que requiere habilidades en estrategia. Se necesita una comprensión total de la organización y de la información de carácter sistémico, debido a las implicaciones de las predicciones sobre el agregado de la compañía.

Sin embargo, no bastan los conocimientos desde lo instrumental para el abordaje de una herramienta planteada desde la estrategia, ya que uno de sus principales retos radica precisamente en su propio diseño. Para esto se requiere una mayor formación interdisciplinar, como señala Gómez (2003) respecto a las potencialidades de investigación en Colombia. Por lo tanto, el investigador debe estar capacitado con herramientas de "análisis de la sociología, la economía política, la ecología, el derecho y las perspectivas heterodoxas de la administración de organizaciones y de la economía, entre otras, unido con las metodologías de ciencias sociales" (p. 144). 
Es interesante analizar esta relación, ya que puede existir una tendencia a vincular las necesidades de interdisciplinariedad en la educación contable con temas de reflexión en torno al conocimiento. Sin embargo, el forecasting, como instrumento de evaluación, presenta un alto componente pragmático que da un grado de conocimiento interdisciplinar para ser aprovechado en mayor valía. Esta condición corresponde a que dentro de los aspectos metodológicos del forecasting, se encuentra la necesidad combinar diferentes tipos de información y de realizar simulaciones hacia diferentes periodos.

Orozco y Velásquez (2013) destacan la utilidad de la combinación de pronósticos mediante el uso de distintos modelos para predecir el comportamiento futuro de una variable, el uso del mismo modelo con distinta configuración interna, o el uso del mismo modelo con distintos valores de los parámetros. Dentro de la información por combinar, se puede citar: datos sectoriales con datos internos, datos reales con proyecciones, datos actuales con datos anteriores, datos macroeconómicos, tendencias, volumen, cantidades, porcentajes, indicadores de gestión, entre otros.

La combinación de criterios no solo va desde los datos generados, también se encuentra determinada por el cumplimiento de los objetivos, lo cual arroja los siguientes interrogantes para el abordaje de estas herramientas: ¿cuál es el nivel óptimo de proyección?, ¿hasta dónde es pertinente crear un escenario basado en supuestos que en el ciclo económico se debe convertir en real, con niveles de variación que deben acercarse a 0 ?

Marquez y Ericcson (1998) aportan un marco tripartito para generar e interpretar pronósticos económicos fundamentado en tres pasos: 1) se definen las variables y se diseña el modelo para realizar el forecasting; 2i) se corre el modelo, se obtiene la información y se evalúa; y, por último, 3) se realiza un análisis post-evaluación, el cual tiene como objetivo generar retroalimentación a partir de la información obtenida, fijando la atención en aspectos tales como el error, es decir, la diferencia entre el valor pronosticado y el valor realmente obtenido. 
Esta multiplicidad de factores genera otro tipo de requerimientos adicionales a la instrumentalización contable y la interdisciplinariedad. El diseño en sí mismo depende de la capacidad de relacionar conceptos desde diferentes áreas. Lo anterior, teniendo en cuenta que dependiendo de la intención del informe, se puede establecer el número de variables deseadas para analizar y la valoración de los efectos sobre los resultados futuros de la organización.

Si la intencionalidad de estas herramientas depende de su diseño, y si esta información puede llegar a ser implementada por la organización internamente, también puede tener trascendencia sobre usuarios externos, por ejemplo, datos de forecasting aplicados al análisis de flujo de caja para futuros inversionistas.

\section{Ética en la toma de decisiones}

Luego de las anteriores consideraciones, el siguiente elemento por tener en cuenta es el factor ético en las decisiones de estructuración de herramientas de evaluación a través de pronósticos, ya que a partir de ellas se pueden determinar directrices como reducciones o aumentos de personal, cambio de insumos, alteración de las estrategias comerciales, entre otras.

Un punto recurrente al tratar el tema ético es la credibilidad, ya que moverse en el campo de las suposiciones y las predicciones es complejo. Es en este caso dónde se retoma el primer elemento mencionado desde la contabilidad estratégica: la cultura organizacional y su vínculo con los valores individuales de cada miembro de la organización.

Ghosal (2005) (citado en Ruíz, Ruíz y Martínez, 2012) evidencia una inquietud respecto al papel de la formación profesional desde el ámbito académico, ya que se ve permeada por una marcada relevancia hacia la consideración de empresa desde la perspectiva de mercado, desencadenando una cultura de egoísmo con negativas consecuencias. Para Demski (2007), la formación universitaria en materia contable se está convirtiendo 
en preparación para el primer empleo, relegando a un lugar secundario los aspectos clave que deberían ser estudiados por los estudiantes de contabilidad, tales como la valoración, los problemas que se presentan en la preparación y auditoría de la información, entre otros.

Nussbaum (2010) plantea que la preferencia y orientación de los estudiantes y de las universidades por la formación en economía y finanzas, en aras de aportar a la productividad y generación de riqueza por parte de las organizaciones, está relegando a un segundo plano la importancia que tiene en los procesos de formación el estudio de las humanidades, ya que estas estimulan y fortalecen el pensamiento, la creatividad y la imaginación.

Al respecto, se podría pensar que la ética empresarial puede vincularse como generadora de valor, lo cual depende de la cultura organizacional. Vale la pena preguntarse si las herramientas de control y planeación que conocemos y que generamos desde la estrategia responden en un alto porcentaje a los valores éticos de la compañía articulados con el individuo.

Este requerimiento desde la cultura de las organizaciones fue revisado por Waddock y Graves (citados en Ruíz et al., 2012, p. 2), al señalar que existe una motivación por parte de la empresa en demostrar un comportamiento ético como estrategia empresarial, la cual se traduce en un mayor reconocimiento y aceptación por parte de los grupos de interés, además de una mejora de la reputación de la empresa y mayores resultados financieros positivos.

Sin embargo, el trabajo de Ruíz (et al., 2012) plantea el abordaje desde una segunda perspectiva, en la que se defiende el valor derivado de la ética organizacional. Esta parte del deseo de un comportamiento ético en la empresa, el cual tiene valor por sí mismo, en tanto que ayudar a un individuo a ser ético supone conducirle a la excelencia, la cual se reflejará en un conjunto de comportamientos positivos desde lo organizacional.

El impacto que una cultura organizacional ética tiene sobre la generación de valor sostenible para las empresas constituye un elemento que se 
debe revisar desde la conceptualización de las estrategias de gestión y su vínculo con la generación de información. Ruíz (et al., 2012) proponen que las "decisiones que se tomen en relación a la implementación de la ética en las organizaciones y que impliquen la disposición y el desarrollo de factores controlables de naturaleza organizacional, repercutirán en la generación de valor empresarial" (p. 4).

De acuerdo con lo anterior, existe una importancia de los recursos intangibles, dentro de los cuales se encuentran conocimientos, capacidades y habilidades tales como la posibilidad de generar valor a través del trabajo, elemento que abordan Siegel y Sorensen (1999).

Se plantean dos proposiciones básicas para entender la ética como motor de una cultura organizacional sostenible, que debería ir asociada al abordaje de las herramientas de evaluación desde lo estratégico:

Proposición 1: "Una cultura organizacional ética generará valor a través de mejorar la respuesta laboral del empleado" (Ruíz et al., 2012, p. 7). Esta proposición se explica desde cómo se influye positivamente sobre el empleado de la siguiente forma: grado de satisfacción, nivel de compromiso, intención de permanecer en la empresa y civismo organizacional.

Proposición 2: "El grado de ajuste de valores 'empresa-empleado' media parcialmente la relación existente entre una cultura organizacional ética y la respuesta laboral del empleado". Este planteamiento integrador propone la creación de valor como un resultado de la combinación de sus dos proposiciones, desde las cuales la perspectiva de la organización en el comportamiento ético se acoge a la visión aristotélica desde la cual es el comportamiento ético el que genera valor como resultado de la interacción en sí misma. No es el resultado de una estrategia de posicionamiento de imagen y reputación, como lo plantean otras teorías de estrategia empresarial.

Resulta adecuado para los contadores públicos de hoy en día el dominio de estas herramientas, ya que estas les permitirán apoyar la gestión, y 
con ello tener un rol más participativo y de mayor relevancia en las áreas directivas y estratégicas de las organizaciones.

Lo anterior se logra mediante el estudio y aprendizaje de diversos desarrollos estadísticos y econométricos, pero comprendidos en el contexto de la toma de decisiones organizacionales en ambientes de riesgo e incertidumbre. El objetivo entonces es determinar las posibilidades y limitaciones que estas técnicas pueden brindar desde una perspectiva estratégica.

De acuerdo con los elementos expuestos desde la ética y desde lo instrumental, los requerimientos del forecasting, desde la perspectiva de la estrategia, se circunscriben en lo que podríamos denominar el diálogo común de saberes. Por ejemplo, Rosaldo (2004) resalta la necesidad de la interdisciplinariedad, la cual representa, además, la construcción de un diálogo significativo entre diferentes profesiones, por lo cual no solamente abarcaría una colaboración desde cada área, sino también una necesidad de formación de cada profesional para poder efectuar el ejercicio de conectividad.

Desde las necesidades de instrumentalización propias del forecasting, existen algunas técnicas y herramientas estadísticas y econométricas que ayudan a la realización de esos pronósticos. El dominio de estas herramientas no solo se convierte en un referente, sino también en una necesidad desde la formación del profesional. A continuación se realiza una breve descripción de las principales.

\section{Análisis de series de tiempo}

Levin y Rubin (2010) afirman que el análisis de series de tiempo es una herramienta econométrica que tiene como objetivo la realización de análisis sobre datos históricos, con el fin de identificar patrones de comportamiento y tendencias, y a partir de allí, elaborar estimaciones sobre el comportamiento futuro de las variables objeto de estudio. 
En este contexto, plantean los mismos autores, resulta adecuado el estudio de los diferentes cambios que se puedan presentar en las condiciones del mercado, tales como fluctuaciones cíclicas - los cambios que se presentan en el ciclo económico-, variaciones estacionales — patrones identificables que se repiten periódicamente, tales como el consumo de elementos de papelería al inicio y mitad del año, o los productos de navidad en el último trimestre del año- y cambios irregulares — cambios impredecibles, como los que estamos presenciando actualmente con la fuerte caída en el precio del petróleo y el acentuado aumento del dólar en los últimos meses-.

Identificar el tipo de tendencia que se deriva del análisis de los datos históricos combina la herramienta desde lo instrumental con una categoría interpretativa, ya que si bien estas pueden ser lineales, también pueden ser curvilíneas - como logarítmicas, exponenciales, potenciales y polinómicas-. Ejemplos de ello pueden ser el nivel de contaminación y el ciclo de vida de los productos, tales como los tecnológicos.

\section{Regresión lineal simple}

Esta técnica econométrica tiene como objetivo la predicción de una variable dependiente, a partir del análisis de la información correspondiente a una variable independiente. La forma general de la regresión lineal simple es la siguiente:

$$
\hat{\mathrm{Y}}=\mathrm{a}+\mathrm{bX}
$$

$\hat{Y}=$ Valor estimado de la variable dependiente

$\mathrm{X}=$ Variable independiente

$\mathrm{a}=$ Ordenada al origen, corte con eje $\mathrm{Y}$, valor de $\hat{\mathrm{Y}}$ cuando $\mathrm{X}=0$

$\mathrm{b}=$ Pendiente de la recta de tendencia 
La forma de calcular los valores de a y b, es a través de las siguientes ecuaciones:

$$
\mathrm{b}=\frac{\sum \mathrm{XY}-\mathrm{nX} \hat{Y}}{\sum \mathrm{X}^{2}-\mathrm{nX} \mathrm{X}^{2}}=\hat{\mathrm{Y}}-\mathrm{b} \hat{\mathrm{Y}}
$$

$\mathrm{Y}=$ Observaciones de la variable dependiente

$\mathrm{X}=$ Observaciones de la variable independiente

$\hat{\mathrm{Y}}=$ Media de las observaciones de la variable dependiente

$\mathrm{X}=$ Media de las observaciones de la variable independiente

$\mathrm{n}=$ Número de datos en la serie de tiempo

$\mathrm{a}=$ Ordenada al origen

$\mathrm{b}=$ Pendiente de la recta

Con base en las anteriores ecuaciones, se obtiene la recta de mejor ajuste - aquella que minimiza los errores - permitiendo describir los datos analizados (Levin y Rubin, 2010).

\section{Regresión lineal múltiple}

Similar a la regresión lineal simple, pero en este caso, al momento de pronosticar una variable dependiente, se tiene en cuenta la información de más de una variable independiente. La siguiente ecuación representa el modelo a seguir:

$$
\hat{Y}=a+b X+c W+d Z+E
$$

Conforme a esta fórmula, $X, W$ y $Z$ serían las variables independientes útiles para predecir el valor de $\hat{Y}$, y $E$ sería el error propio al modelo. Los 
coeficientes b, cy d representan la capacidad que tiene la respectiva variable independiente de explicar los cambios en la variable dependiente.

\section{Valor esperado (E)}

Esta técnica de pronóstico tiene como objetivo predecir el comportamiento futuro de una variable, teniendo en cuenta la probabilidad de que se presenten los distintos resultados posibles. El valor esperado de la variable $V(E)$ será la sumatoria de los productos de cada posible valor que pueda tomar la variable, multiplicado por su nivel de probabilidad.

\section{Ganancias condicionales}

Esta técnica tiene como objetivo establecer una relación entre los pagos y beneficios que se puedan derivar de una determinada acción. Es muy utilizada para tomar decisiones de inventarios. El criterio de decisión se fundamenta en la relación costo-beneficio, donde se recomienda optar por la acción que genere la mayor brecha entre estos dos factores.

También se pueden encontrar las técnicas de MAXIMIN y MINIMAX, según las cuales las decisiones se deben tomar a partir de maximizar el resultado menos optimista y minimizar la máxima pérdida posible (Newbold, 1998), el esquema de decisión puede analizarse en la figura 1, que se encuentra a continuación.

Hasta este punto se puede señalar que una de las principales necesidades desde la formación se encuentra conectada con la posibilidad de profundizar en conocimientos sobre estadística, matemáticas y economía, para poder asegurar la participación en la construcción, diseño y trazabilidad del forecasting. Esta presunción requiere la exploración, por parte del profesional, de fuentes bibliográficas que le permitan tener mayor acceso a este tipo de herramientas. 
Figura 1. Herramientas para construir pronósticos

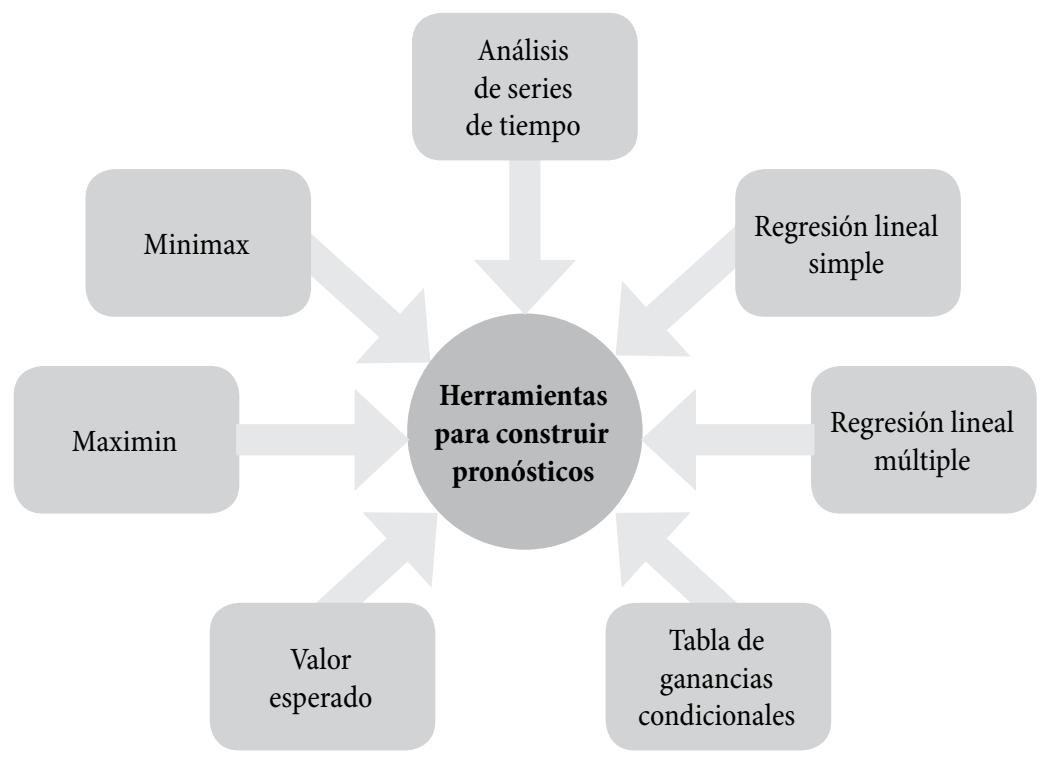

Fuente: elaboración propia.

Al respecto, cabe señalar que el tema del forecasting se ha trabajado desde hace algún tiempo en publicaciones especializadas, las cuales van tomando mayor relevancia dentro del lenguaje empresarial. Estas publicaciones cuentan con mayor consulta y publicación en Norte América y Europa; todavía se observa un rezago en cuanto al pronunciamiento académico y empresarial sobre esta herramienta en América Latina. Igualmente resulta adecuado precisar que al momento de revisar artículos sobre forecasting, la mayoría se refieren a aplicaciones en el campo de las finanzas. La tabla 2 presenta algunas publicaciones sobre forecasting, identificadas en una revisión de bases de datos. 
Tabla 2. Publicaciones académicas sobre forecasting

\begin{tabular}{|c|c|c|c|}
\hline Nombre & Tipo de publicación & Frecuencia & Editor \\
\hline $\begin{array}{l}\text { Advertising } \\
\text { Forecasting }\end{array}$ & $\begin{array}{l}\text { Publicación periódica } \\
\text { sobre comercio }\end{array}$ & Trimestral & $\begin{array}{l}\text { World Advertising } \\
\text { Research Center }\end{array}$ \\
\hline $\begin{array}{l}\text { Blue Chip Financial } \\
\text { Forecast }\end{array}$ & $\begin{array}{l}\text { Publicación periódica } \\
\text { sobre comercio }\end{array}$ & Mensual & $\begin{array}{l}\text { Wolters Kluwer } \\
\text { Law \& Business }\end{array}$ \\
\hline $\begin{array}{l}\text { Dow Theory } \\
\text { Forecasts }\end{array}$ & $\begin{array}{l}\text { Publicación periódica } \\
\text { sobre acciones y } \\
\text { mercado de acciones }\end{array}$ & Mensual & $\begin{array}{l}\text { Horizon } \\
\text { Publishing } \\
\text { Company LLC }\end{array}$ \\
\hline $\begin{array}{l}\text { Foresight: The } \\
\text { International } \\
\text { Journal of Applied } \\
\text { Forecasting }\end{array}$ & $\begin{array}{l}\text { Revista académica } \\
\text { sobre pronósticos y } \\
\text { predicciones }\end{array}$ & Trimestral & $\begin{array}{l}\text { International } \\
\text { Institute of } \\
\text { Forecasters }\end{array}$ \\
\hline $\begin{array}{l}\text { International } \\
\text { Journal of } \\
\text { Forecasting }\end{array}$ & $\begin{array}{l}\text { Revista académica } \\
\text { sobre pronósticos }\end{array}$ & Trimestral & $\begin{array}{l}\text { Elsevier Science } \\
\text { Limited }\end{array}$ \\
\hline $\begin{array}{l}\text { Journal of Business } \\
\text { Forecasting }\end{array}$ & $\begin{array}{l}\text { Revista académica } \\
\text { sobre pronóstico } \\
\text { económico }\end{array}$ & Trimestral & $\begin{array}{l}\text { Graceway } \\
\text { Publishing } \\
\text { Company }\end{array}$ \\
\hline $\begin{array}{l}\text { Journal of Business } \\
\text { Forecasting Methods } \\
\text { \& Systems }\end{array}$ & $\begin{array}{l}\text { Revista académica } \\
\text { sobre negocios, } \\
\text { pronósticos y } \\
\text { predicciones }\end{array}$ & Trimestral & $\begin{array}{l}\text { Graceway } \\
\text { Publishing } \\
\text { Company }\end{array}$ \\
\hline Journal of Forecasting & $\begin{array}{l}\text { Revista académica } \\
\text { sobre pronóstico y } \\
\text { predicción }\end{array}$ & Bimestral & Wiley-Blackwell \\
\hline $\begin{array}{l}\text { Forecasts for } \\
\text { Management } \\
\text { Decisionmaking }\end{array}$ & $\begin{array}{l}\text { Publicación periódica } \\
\text { sobre Estados Unidos, } \\
\text { política económica y } \\
\text { pronóstico económico }\end{array}$ & Mensual & $\begin{array}{l}\text { Kiplinger } \\
\text { Washington } \\
\text { Editors }\end{array}$ \\
\hline $\begin{array}{l}\text { Perryman Long-Term } \\
\text { Economic Forecast }\end{array}$ & $\begin{array}{l}\text { Publicación periódica } \\
\text { sobre pronóstico } \\
\text { económico }\end{array}$ & Anual & Perryman Group \\
\hline $\begin{array}{l}\text { Romanian Journal of } \\
\text { Economic Forecasting }\end{array}$ & $\begin{array}{l}\text { Revista académica } \\
\text { sobre pronóstico } \\
\text { económico }\end{array}$ & Trimestral & $\begin{array}{l}\text { The Institute } \\
\text { for Economic } \\
\text { Forecasting }\end{array}$ \\
\hline
\end{tabular}

Fuente: elaboración propia. 
También existen algunos software especializados que ayudan al proceso de pronóstico. Siguiendo a Diebold (1999), estos son los más conocidos y utilizados:

- Eviews

- $\mathrm{S}+$

- SAS

- Minitab

- Matlab

- Stata

- RATS

\section{Conclusiones}

La apropiación del discurso en torno a la estrategia e instrumentos como el forecasting y la disciplina contable desde lo pedagógico han mostrado transformaciones no solo desde los aspectos de contenido y metodológicos, sino también desde la construcción de un diálogo significativo entre saberes.

Desde el conocimiento contable se generan unas necesidades para el abordaje de herramientas como el forecasting, determinadas por la capacidad de vincular conocimiento instrumental e interdisciplinar con las prácticas de gestión estratégica de las organizaciones.

Si la intencionalidad de estas herramientas depende de su diseño, y si esta información puede llegar a ser implementada por la organización internamente, también pueden tener trascendencia sobre usuarios externos - por ejemplo, datos de forecasting aplicados al análisis de flujo de caja para futuros inversionistas-. Tal situación nos introduce en un debate 
ético acerca de la información generada por las organizaciones y el uso que se hace de esta.

Esta multiplicidad de factores genera otro tipo de requerimientos adicionales a la instrumentalización contable y la interdisciplinariedad. El diseño en sí mismo depende de la capacidad de relacionar conceptos desde diferentes áreas. Lo anterior, teniendo en cuenta que dependiendo de la intención del informe, se puede establecer el número de variables deseadas para analizar, y la valoración de los efectos sobre los resultados futuros de la organización.

El pronunciamiento desde la academia para efectos de este tipo de herramientas en América Latina requiere mayor estudio e introspección desde la formación y el ejercicio profesional. Actualmente la mayoría de publicaciones se encuentran ubicadas en Estados Unidos y Europa.

Los requerimientos académicos desde la generación de forecasting se encuentran vinculados a temas de valoración y control. Sin embargo, la necesidad de fortalecer conocimientos en áreas como la estadística, las matemáticas y la economía es un asunto primordial. 


\section{Referencias bibliográficas}

Adelman, M. (1946). Correlations and forecasting. The American Economic Review. 36(4), 645-650.

Artigas, M. (2001). Mi visión de la interdisciplinariedad. Disertación en el seminario del Grupo de Estudios Peirceanos, Universidad de Navarra, Pamplona. Ballou, B., Heitger, D., \& Stoel, D. (2011, verano). How boards of directors perceive risk management information. Management Accounting Quarterly, 12(4), 14-22.

Bauman (2013) Sobre la Educación en un Mundo Líquido: conversaciones con Richard o Mazzeo. Editorial: PAIDOS IBÉRICA, Madrid.

Brewer, P. (2008). Redefining management accounting: Promoting the four pillars of our profession. Strategic Finance, 27-34.

Cable, R. Healy, P. \& Mathew, E. (2009). Teaching future management accountants. Management Accounting Quarterly 10(4), 44-50.

Castillo, J. (1997). El paradigma perdido de la interdisciplinariedad: Volver a los clásicos. Política y Sociedad. Nro. 26, 143-155.

Courtney, H. Kirkland, J. \& Viguerie, P. (1997). Estrategia en tiempos de incertidumbre. En Harvard Business Review (Ed.) La gestión en la incertidumbre (pp. 1-36). Bilbao: Deusto.

Dean, J. (1946). Cost forecasting and price policy. Journal of Marketing. 13(3), pp. 279-288.

Demski, J. (2007). Is accounting an academic discipline? Accounting Horizons 21(2), 153-157.

Díaz. E. \& Suárez, J. (Eds.). (2014). Aproximación conceptual al forecasting en el contexto de la toma de decisiones bajo incertidumbre. En Memorias del I Congreso Colombiano de Costos y Gestión. Bogotá. Universidad Javeriana.

Diebold, F. (1999). Elementos de pronósticos. México: International Thompson.

Ericsson, N. \& Marquez, J. (1998). A framework for economic forecasting. The Econometrics Journal, 1(1), C228-C266.

Fama, E. \& French, K. (2000). Forecasting profitability and earnings. The Journal of Business 73(2), 161-175.

Ghosal (2005). Bad management theories Are Destroying Good Management Practices. Academy of Management Learning \& Education. Vol. 4, Nro. 1; pp. 75-91. 
Glover, J. Ijiri, Y. Levine, C. \& Liang, P. J. (2008). Separación de los hechos y pronósticos en los estados financieros. Innovar, 18(31), 135-146.

Gómez, M. (2003, enero-junio). Algunos comentarios sobre la potencialidad de la investigación en contabilidad. Innovar, (21), 139-144.

Hamel, G. \& Prahalad, C. (1994). Compitiendo por el futuro. En Harvard Business Review (Ed.). La gestión en la incertidumbre. Bilbao: Deusto, pp. 37-56.

Hanke, J. \& Wichern, D. (2006). Pronósticos en los negocios. México: Pearson.

Harvard Business Review (Ed.). (1999). La gestión en la incertidumbre. Bilbao: Deusto.

Horngren, D. \& Foster. E. (2007). Contabilidad de costos: Un enfoque gerencial. México: Pearson.

Howard, J. (1954). A note on corporate forecasting practices. The Journal of Business, 27(1), 101-105.

Jiménez, R. (2013). Aproximación a la teoría de la decisión: Organizaciones y contabilidad. Lúmina, nro. 8, 108-122.

Levin, R., \& Rubin, D. (2010). Estadística para administración y economía. México: Pearson Educación.

Makridakis, S. Hibon, M. \& Moser, C. (1979). Accuracy of forecasting: An empirical investigation. Journal of the Royal Statistical Society. 142(2), 97-145.

Marie, A. Cheffi, W. Jean, R. \& Rao, A. (2010). Is standard costing still relevant? Management Accounting Quarterly, 11(2), 1-10.

Marquez y Ericcson (1998). A framework for Economic Forecasting. The Econometrics Journal, vol 1, Issue 1. Pp.203-227.

Mathew, E. (2009). Teaching future management accountants. Management Accounting Quarterly, 10(4), 44-50.

Newbold, P. (1999). Estadística para los negocios y la economía. Madrid: Prentice Hall.

Nussbaum, M. (2010). Sin fines de lucro: Por qué la educación necesita de las humanidades. Buenos Aires: Katz.

Orozco, J. \& Velásquez, J. (2013, octubre-diciembre). A new forecasting combination system for predicting volatility. Innovar. Revista de Ciencias Administrativas $y$ Sociales, 23(50), 5-26.

Rosaldo, R. (2004). Reflexiones sobre la interdisciplinariedad. Revista de Antropología Social, nro. 13, 197-215. 
Ruíz, P. Ruíz, C. \& Martínez, R. (2012, enero-abril), Cultura organizacional ética y generación de valor sostenible. Investigaciones Europeas de Dirección y Economía de la Empresa, 18(1), 017-031.

Shahzad, L. \& Shabbir, K. (2012). Impact of organizational culture on organizational performance: An overview. Interdisciplinary Journal of Contemporary Research in Business, 3(9), 975-985.

Siegel, S. \& Sorensen, A. (1999,). Counting more, counting less transformations in the management accounting profession. Practice Analysis of Management Accounting, 39-44.

Wagle, B. (1969). Environmental forecasting and corporate planning. Economic and Political Weekly, 4(22), M37, M39-M42.

Walker, R. (1929). Business forecasting. The Australian Quarterly, 1(3), 101-109.

Weston, J. (1958). Forecasting financial requirements. The Accounting Review, 33(3), pp. 427-440.

Weston, J. (1958). Forecasting financial requirements. The Accounting Review, 33(3), pp. 427-440. 